\title{
Mensuração de Coping no Ambiente Ocupacional
}

\author{
Fernanda Amaral Pinheiro ${ }^{1}$, \\ Bartholomeu Tôrres Tróccoli e \\ Mauricio Robayo Tamayo \\ Universidade de Brasília
}

\begin{abstract}
RESUMO - Coping $^{2}$ pode ser definido pela forma como as pessoas comumente reagem ao estresse. Estas reações estão relacionadas a fatores pessoais, demandas situacionais e recursos disponíveis (Lazarus \& Folkman, 1984). Entretanto, medidas de coping geral raramente contemplam os fatores situacionais. A mensuração de coping no ambiente ocupacional deve considerar os recursos e estratégias disponíveis, permitir agilidade ao preenchimento e satisfazer critérios psicométricos usuais. O objetivo deste trabalho foi (1) traduzir e adaptar para a língua portuguesa uma escala de coping ocupacional; (2) investigar suas características psicométricas por meio de análise fatorial e por suas relações com medidas de suporte social, sobrecarga de trabalho e exaustão emocional. Trezentos e noventa e sete trabalhadores em ambiente de escritório $(\mathrm{x}=37,9 ; \mathrm{dp}=9,7)$ responderam à escala traduzida e a medidas de suporte social, sobrecarga e exaustão emocional durante o expediente de trabalho. A análise fatorial mostrou a existência de três fatores que explicaram $29,6 \%$ da variância total. Os resultados forneceram evidências de validade de critério e confiabilidade à escala de coping ocupacional traduzida.
\end{abstract}

Palavras-chave: estresse ocupacional; coping; medida psicológica.

\section{Coping Measurement in Occupational Setting}

\begin{abstract}
Coping represents the way people commonly react to stress. These reactions are related to personal factors, situational demands and available resources (Lazarus \& Folkman, 1984). However, measures of general coping rarely fully contemplate situational factors. Coping measures in occupational settings should take into account available resources, should be easy to answer and fulfil all psychometric criteria. The objective of this work was (1) to translate and to adapt to Portuguese an occupational coping scale and (2) to investigate its psychometric characteristics through factor analysis and its relationships with measures of social support, work overload and emotional exhaustion. Three hundred ninety seven office workers $(\mathrm{x}=37.9$; $\mathrm{sd}=9.7)$ answered in their workplace the proposed occupational coping scale and measures of social support, work overload and emotional exhaustion. Factor analyses showed three factors that explained $29.6 \%$ of the total variance. The results gave support to the criterion validity and reliability of the translated occupational coping scale.
\end{abstract}

Key words: occupational stress; coping; psychological measure.

Define-se coping como uma variável individual representada pelas formas como as pessoas comumente reagem ao estresse, determinadas por fatores pessoais, exigências situacionais e recursos disponíveis (Lazarus \& Folkman, 1984).

A pesquisa na área de coping tem se mantido bastante produtiva nas últimas duas décadas. Apesar disto, questões conceituais e metodológicas envolvidas no estudo do construto permanecem sob discussão.

Um aspecto comumente ressaltado entre os estudos diz respeito à falta de consenso sobre os tipos de estratégias de coping a serem avaliados. Diversos autores têm utilizado uma taxonomia de coping relativa ao foco de atenção do indivíduo: na solução de problemas ou na regulação da emoção envolvida (Carver, Scheier \& Weintraub, 1989; Cohen, 1987; Folkman \& Lazarus, 1980). Atualmente, esta distinção tem sido questionada por seu fraco poder explicativo do comportamento (Dewe, Cox \& Ferguson, 1993).

1 Endereço: SHIN QI 02, conjunto 13, casa 04 - Lago Norte Brasília/DF - 70510-130. E-mail: fernandaamaral@ terra.com.br

2 O termo coping será mantido em inglês devido ao fato de a palavra enfrentamento, por vezes utilizada como tradução, induzir a pensar em formas ativas de lidar com o estresse, o que excluiria outras formas como a esquiva, por exemplo.
Outra distinção comum entre os estudos diz respeito às diferentes categorias gerais de estratégias utilizadas pelos indivíduos como formas de enfrentar o estresse, tais como busca de informações, ação direta, inibição da ação, processos intrapsíquicos e busca de apoio social (Carver \& cols., 1989; Dewe \& cols., 1993; Taylor, 1986). Em muitos estudos, as opções de estratégias têm sido reduzidas a um número menor de dimensões, como a busca de controle e a esquiva.

Uma segunda questão que permanece sob discussão e está intrinsecamente relacionada ao problema conceptual é a questão da mensuração do construto. De fato, uma variedade de medidas tem sido utilizada, algumas delas sem a devida avaliação quanto às propriedades psicométricas (Latack, 1986). Dentre as medidas mais usadas, a Ways of Coping - WOC, versão atualizada da Ways of Coping Checklist (Folkman, Lazarus, Dunkel-Schetter, DeLongis \& Gruen, 1986) é a mais citada, além de servir como base para a construção de outras escalas. Outras medidas que têm recebido atenção na literatura especializada são a Coping Strategy Indicator - CSI (Amirkhan, 1990) e a COPE (Carver \& cols., 1989). Estas medidas procuram verificar o grau em que as pessoas utilizam cada uma das estratégias definidas pelos modelos teóricos subjacentes em seu dia a dia, sendo, portanto, consideradas medidas de coping geral. 
Para a investigação sobre estratégias de coping utilizadas pelos indivíduos em situações específicas, como no caso do acometimento de doenças crônicas ou frente a problemas típicos do ambiente de trabalho, o pesquisador deve incluir às escalas de coping geral estímulos ou episódios, que são descrições de situações que levam o respondente a se reportar ao contexto desejado. Entretanto, dada a importância da dimensão situacional na própria definição de coping, medidas desenvolvidas para situações específicas são sempre bem vindas por apresentarem estratégias que realmente se aplicam ao contexto em questão. Em reforço a este argumento, Parkes (1990) ressalta que algumas estratégias de coping que são consideradas efetivas em ambiente doméstico e no relacionamento conjugal não têm se mostrado capazes de aliviar o estresse no contexto ocupacional, ou conseguem fazê-lo apenas em uma extensão limitada. Uma possível razão para este fato é que as limitações inerentes ao contexto ocupacional restringem as possibilidades para a ação efetiva dos indivíduos, que passam a depender de diferentes estratégias de coping. Além disso, há peculiaridades metodológicas que precisam ser consideradas. No caso específico do ambiente de trabalho, as escalas deveriam, por exemplo, permitir agilidade ao preenchimento.

Dewe, Cox e Ferguson (1993) também defenderam o desenvolvimento de escalas que mensurassem coping no ambiente ocupacional, alegando que, se por um lado, há perda de comparabilidade entre as situações, por outro, há a possibilidade de se capturar a influência específica da cultura da organização ou de determinado comportamento de um grupo ocupacional. Aqueles autores alertaram para dificuldades envolvidas no desenvolvimento de escalas de coping baseadas apenas em estudos empíricos que, a partir de questões abertas ou semi abertas, levantam uma série de itens e deixam que a análise fatorial faça "o resto do trabalho". Revendo a literatura a respeito do tema, eles relataram que os estudos mais adequados oferecem uma análise detalhada da literatura para a seleção dos itens sobre a qual desenvolvem hipóteses a serem testadas. Um exemplo de escala que deriva de estudos deste tipo seria a medida desenvolvida por Latack (1986).

Em seu modelo para a compreensão de coping ocupacional, Latack (1986) considerou que ações e reavaliações cognitivas deveriam estar, juntas, relacionadas a estratégias de enfrentamento ou de esquiva da situação estressante. Às ações e reavaliações cognitivas relacionadas ao enfrentamento, a autora nomeou estratégias de controle, enquanto que ações e reavaliações cognitivas de conteúdo escapista foram chamadas estratégias de esquiva. A autora também incluiu em sua classificação as estratégias de manejo de sintomas para denominar as tentativas de lidar com os sintomas de estresse popularmente aceitas, como o relaxamento ou a prática de exercícios físicos.

Coping tem mostrado relações significativas com variáveis demográficas como gênero (Parkes, 1990), medidas de diferenças individuais como locus de controle (Parkes, 1984), neuroticismo e depressão (Endler \& Parker, 1990), afetividade negativa (Parkes, 1990) e saúde (Cohen, 1987). Folkman e Lazarus (1980) relataram maior utilização, no ambiente ocupacional, de estratégias voltadas para a focalização do problema. Em seu estudo com 100 sujeitos, os autores também verificaram diferenças significativas na utilização das estratégias de coping ocupacional quanto ao gênero (homens utilizaram mais estratégias que focalizam o problema do que mulheres), embora o tipo de ocupação dos sujeitos não tenha sido controlado. Não foram verificados efeitos em coping relacionados à idade.

Relações interativas entre coping, estressores no trabalho e efeitos sobre a saúde e o desempenho nas organizações também têm sido relatadas (Parkes, 1986, 1990 e 1994). Em um estudo desenvolvido com 135 estudantes de enfermagem, Parkes (1986) encontrou relações indiretas entre controle, neuroticismo e sobrecarga: sujeitos que apresentaram altos escores em neuroticismo mostraram relação curvilinear (em forma de U invertido) entre sobrecarga e controle, de forma que altos ou baixos níveis de sobrecarga estiveram associados à menor utilização de estratégias de controle. Este padrão não se verificou para sujeitos com baixos escores em neuroticismo. Em um outro estudo (Parkes, 1990), a mesma autora relatou efeitos diretos e indiretos de coping sobre uma medida de saúde mental (escores do General Health Questionnaire - GHQ). Supressão, uma forma de coping semelhante à esquiva, mostrou efeito direto sobre os escores do GHQ, onde escores mais altos significam maior nível de estresse. Controle mostrou efeito indireto em saúde mental, moderando a relação entre percepção de demandas no trabalho e escores do GHQ, de forma que indivíduos que faziam mais uso de estratégias de controle (mais um desvio padrão) não mostraram associação entre demandas no trabalho e efeitos sobre a saúde mental, enquanto que sujeitos com baixa utilização de controle (menos um desvio padrão) mostraram associação significativa entre aquelas variáveis. O mesmo efeito indireto foi encontrado entre controle, suporte social no trabalho e escores do GHQ, mostrando que a utilização da estratégia reduziu o efeito negativo da falta de suporte social sobre a saúde mental.

$\mathrm{O}$ trabalho de que trata este artigo diz respeito à tradução e à avaliação psicométrica da versão brasileira da escala desenvolvida por Latack para mensurar coping no ambiente ocupacional. Para tal, buscou evidências de validade por meio da verificação da estrutura fatorial da escala traduzida e do padrão de relações estabelecidas com algumas variáveis descritas na literatura como correlacionadas a estilos de coping (suporte social, sobrecarga de trabalho e exaustão emocional).

\section{Método}

\section{Sujeitos}

Participaram deste estudo 396 empregados de cinco empresas brasileiras com sede em Brasília (três estatais e duas da iniciativa privada), onde ocupavam variados cargos. A média de idade foi de 37,3 anos (desvio padrão $=$ 9,7). Do total da amostra, 51,5\% eram mulheres, $43 \%$ dos respondentes tinham escolaridade superior; $60 \%$ eram casados e $67 \%$ tinham jornada de trabalho diária de seis horas. Foram realizados contatos com supervisores de cada uma das empresas, onde se explicou o objetivo da pesquisa, garantiu-se a confidencialidade das informações e solicitou-se a distribuição dos questionários pelo maior número possível 
de sujeitos. A seguir, os empregados foram contatados por seus supervisores e convidados a preencher um questionário que incluiu a escala de coping traduzida, medidas de suporte social e sobrecarga no trabalho (Pinheiro \& Tróccoli, 2001) e uma medida de exaustão emocional (Tamayo \& Tróccoli, 2000). Após o intervalo de uma semana, os pesquisadores retornavam à instituição para recolher os questionários preenchidos.

\section{Instrumentos}

A escala desenvolvida por Latack (1986) foi traduzida para a língua portuguesa e alguns itens sofreram leves alterações, enquanto outros foram reagrupados.

Tendo em vista que as estratégias de coping no estudo original mantiveram-se constantes entre os diferentes tipos de estressores, optou-se por solicitar aos sujeitos que respondessem com base em um único estímulo, abrangente o suficiente para suscitar estratégias utilizadas em geral, sem perder de vista a peculiaridade do ambiente ocupacional. Assim, os itens foram precedidos da seguinte frase: "Quando tenho um problema no trabalho eu ...", seguida da relação de itens.

A validação semântica dos itens foi realizada com cinco indivíduos de nível de escolaridade médio, resultando em pequenas mudanças quanto à forma. Um conjunto de 46 itens abrangendo os três estilos hipotetizados envolveu 17 itens de controle, 11 itens de esquiva e 18 itens de manejo de sintomas e foram, a seguir, apresentados a cinco juizes, especialistas da área do construto, para classificação quanto aos fatores sugeridos por Latack (1986). Cinco itens foram considerados suspeitos por apresentarem concordância menor que $75 \%$ entre os juizes ou porque foram classificados em um fator diferente do proposto pela autora (quase sempre isto se deu entre itens de esquiva e de manejo de sintomas). Dentre os itens suspeitos, três foram excluídos por não apresentarem carga acima do critério de 0,40 em nenhum fator; os outros dois se agruparam conforme o instrumento original sendo, portanto, mantidos.

Escala de Percepção de Sobrecarga no Trabalho (Pinheiro \& Tróccoli, 2001): Desenvolvida como parte de um instrumento para mensurar aspectos psicossociais do ambiente ocupacional antecedentes de doenças osteomusculares (assim como a medida de suporte social, a seguir), a escala incluiu 8 itens e apresentou índice de confiabilidade alpha = 0,84 . Exemplos de itens da escala são: "a minha organização submete o funcionário a uma carga excessiva de trabalho", "meu chefe espera dos seus subordinados mais do que eles são capazes de produzir".

Escala de Percepção de Suporte Social (Pinheiro \& Tróccoli, 2001). Cinco itens compuseram a escala, que exibiu índice de confiabilidade alpha $=0,86$. Exemplos de itens: "meu grupo se preocupa com o bem-estar dos colegas de trabalho", "os colegas colaboram uns com os outros na realização do trabalho".

Escala de Exaustão Emocional: A medida de reatividade ao estresse ocupacional utilizada foi uma subescala do instrumento de medida de exaustão emocional desenvolvida por Tamayo e Tróccoli (2000). Exaustão é considerada a dimensão central de burnout, compreendido como uma reação específica ao estresse ocupacional, e tem sido defi- nida como o sentimento de ser sobrecarregado de trabalho e ser esvaziado de recursos emocionais (Gaines \& Jermier, 1983). A subescala utilizada apresentava 4 itens e índice de confiabilidade alpha $=0,89$ ("sinto-me emocionalmente vazio com o meu trabalho").

Variáveis demográficas: Uma seção adicional do questionário foi destinada a aferir características demográficas e ocupacionais dos sujeitos. As variáveis incluídas foram gênero (feminino $=1$, masculino $=2$ ) e tempo de serviço, em anos.

\section{Resultados}

\section{Estrutura Fatorial da Escala de Coping Ocupacional}

Uma análise prévia dos dados foi realizada para investigar a presença de dados faltosos e casos extremos. Os primeiros foram substituídos pela média das variáveis e os casos extremos multivariados $(\alpha=0,001)$, num total de 27 , foram deletados da planilha, totalizando 369 dados válidos (Tabachnick \& Fidell, 2001). O último item da escala de manejo de sintomas ("fumo mais do que de costume") foi excluído da análise por constituir-se em uma fonte sistemática de dados faltosos, em razão de problemas na diagramação do instrumento.

A matriz de correlações foi considerada fatorializável, apresentando KMO = 0,796 e índice de Bartlett significativo (Pasquali, 1999). Utilizando-se o Método dos Eixos Principais (Principal Axis Factoring - PAF) e rotação Oblimin, 3 fatores foram extraídos, com número adequado de itens compatíveis com os fatores originais controle, esquiva e manejo de sintomas. A maior das correlações entre os fatores foi entre manejo de sintomas e esquiva $(r=0,27 ; p<$ $0,01)$ enquanto a menor ocorreu entre controle e esquiva $(r$ $=0,07 ; p<0,01)$.

As comunalidades, variância explicada, eigenvalues, carga dos itens e índice de confiabilidade alpha de cada fator estão dispostos na tabela 1. Cargas inferiores a 0,40 foram omitidas para facilitar a visualização dos resultados.

Os três fatores explicaram 29,6\% da variância e, utilizando-se como critério a carga mínima de 0,40 , variáveis complexas não foram identificadas. Dezesseis dentre os 45 itens não foram classificados em nenhum dos três fatores. A solução foi considerada satisfatória, apresentando fatores consistentes, com número adequado de itens e boas cargas fatoriais, reproduzindo fielmente a estrutura proposta por Latack (1986). Os índices de confiabilidade interna mostraram-se adequados e variaram entre 0,77 e 0,81 .

\section{Validade de Critério da Escala Traduzida: Relações com Outras Variáveis}

Teste te ANOVA foram utilizados para investigar diferenças entre médias dos grupos. Mulheres apresentaram maiores médias do que homens em manejo de sintomas (2,42 e 2,33 respectivamente) e esquiva (2,41 e 2,33 respectivamente), embora a diferença não tenha alcançado significância estatística. Homens apresentaram maior média em controle do que as mulheres $(3,77$ e 3,73 , respectivamente), e esta diferença 
Tabela 1. Carga fatorial, comunalidades (h2), eigenvalues e percentual de variância para os itens da escala de coping ocupacional.

\begin{tabular}{|c|c|c|c|c|}
\hline \multirow{2}{*}{ Item } & \multicolumn{3}{|c|}{ Fatores } & \multirow{2}{*}{$\mathbf{h}^{2}$} \\
\hline & Manejo & Controle & Esquiva & \\
\hline Tiro alguns dias para descansar & 0,759 & & & 0,641 \\
\hline Faço uma viagem & 0,758 & & & 0,679 \\
\hline Mudo os meus hábitos alimentares & 0,554 & & & 0,372 \\
\hline Compro alguma coisa & 0,536 & & & 0,435 \\
\hline Procuro me envolver em mais atividades de lazer & 0,532 & & & 0,429 \\
\hline Me torno mais sonhador(a) & 0,514 & & & 0,485 \\
\hline Uso algum tipo de técnica de relaxamento & 0,427 & & & 0,390 \\
\hline Procuro a companhia de outras pessoas & 0,415 & & & 0,444 \\
\hline Penso na situação como um desafio & & 0,577 & & 0,469 \\
\hline Decido sobre o que deveria ser feito e comunico ... & & 0,566 & & 0,404 \\
\hline Tento modificar os fatores que causaram a situação & & 0,548 & & 0,387 \\
\hline Dou atenção extra ao planejamento & & 0,505 & & 0,420 \\
\hline Tento ver a situação como uma oportunidade para ... & & 0,495 & & 0,457 \\
\hline Tento trabalhar mais rápida e eficientemente & & 0,466 & & 0,436 \\
\hline Me esforço para fazer o que eu acho que se ... & & 0,455 & & 0,390 \\
\hline Penso em mim como alguém que sempre ... & & 0,453 & & 0,400 \\
\hline Converso com colegas que também estejam ... & & 0,451 & & 0,358 \\
\hline Peço conselho a pessoas que, embora estejam ... & & 0,409 & & 0,319 \\
\hline Tento manter distância da situação & & & 0,708 & 0,496 \\
\hline Mantenho a maior distância possível das ... & & & 0,606 & 0,429 \\
\hline Digo a mim mesmo que o tempo resolve ... & & & 0,600 & 0,417 \\
\hline Procuro lembrar que o trabalho não é tudo na vida & & & 0,534 & 0,355 \\
\hline Antecipo as conseqüiências negativas, ... & & & 0,517 & 0,325 \\
\hline Evito a situação, se possível & & & 0,441 & 0,330 \\
\hline Concentro-me em fazer prioritariamente ... & & & 0,426 & 0,314 \\
\hline Tento não me preocupar com a situação & & & 0,408 & 0,309 \\
\hline Percentual de variância & 13,286 & 10,632 & 5,713 & \\
\hline Eigenvalues & 5,979 & 4,784 & 2,571 & \\
\hline Coeficiente alpha de Cronbach & 0,813 & 0,788 & 0,774 & \\
\hline
\end{tabular}

também não foi considerada estatisticamente significativa. Diferenças em utilização das estratégias de coping devido à escolaridade não foram verificadas.

Análises de regressão logística hierárquica foram conduzidas para investigar possíveis preditores dos três estilos de coping, entre variáveis demográficas, exaustão emocional e demandas no trabalho (sobrecarga e suporte social). A tabela 2 apresenta coeficientes de regressão não padronizados (B), coeficientes padronizados $(\beta)$ e o seu nível de significância $p$; correlações semiparciais (controlando-se o efeito das outras variáveis) entre as variáveis independentes e os três estilos de coping. Os valores da variância explicada pelo conjunto de variáveis com o modelo completo ( $\mathrm{r}^{2}$ cumulativo) e por cada conjunto em particular ( $\mathrm{r}^{2}$ do passo) são também fornecidos.

Dentre as variáveis demográficas, apenas tempo de serviço foi capaz de predizer a utilização da estratégia de controle. A contribuição foi discreta, respondendo por apenas $2 \%$ da variância explicada.
Exaustão emocional foi preditor de controle e esquiva, na direção esperada: a presença de exaustão está negativamente associada a controle e positivamente associada à esquiva.

As demandas ocupacionais estiveram relacionadas a todos os estilos de coping, na direção esperada: a percepção de sobrecarga e de suporte social puderam predizer a utilização da estratégia de controle, sendo suporte social o mais importante preditor; suporte social foi também o único preditor da utilização da estratégia manejo de sintomas, enquanto que escores de sobrecarga puderam predizer a utilização de esquiva.

Módulos interativos foram incluídos ao final do modelo para cada uma das análises de regressão, com o objetivo de replicar estudos que encontraram relações indiretas entre demandas ocupacionais e coping. O módulo que incluiu a interação entre exaustão emocional e percepção de sobrecarga quadrática encontrou significância estatística, $p$ $=0,03$. Outras interações entre demandas ocupacionais e exaustão emocional na predição dos estilos de coping não foram observadas. 
Tabela 2. Resultados de análises de regressão logística hierárquica apresentando preditores de coping, a partir de variáveis demográficas, exaustão emocional e demandas ocupacionais.

\begin{tabular}{|c|c|c|c|c|c|c|}
\hline Variáveis & $\begin{array}{c}\mathrm{r}^{2} \\
\text { cumulativo }\end{array}$ & $\begin{array}{c}\mathrm{r}^{2} \\
\text { do passo }\end{array}$ & $\mathrm{B}^{*}$ & B & $\begin{array}{l}\text { Correlação } \\
\text { semiparcial }\end{array}$ & $\mathrm{p}$ \\
\hline \multicolumn{7}{|l|}{ Controle } \\
\hline Tempo de atividade & 0,02 & 0,02 & 0,01 & 0,15 & 0,15 & 0,004 \\
\hline Exaustão emocional & 0,03 & 0,01 & $-0,09$ & $-0,14$ & $-0,11$ & 0,02 \\
\hline Sobrecarga & & & 0,15 & 0,19 & 0,16 & 0,002 \\
\hline Suporte social & 0,12 & 0,08 & 0,18 & 0,27 & 0,25 & 0,000 \\
\hline \multicolumn{7}{|l|}{ Esquiva } \\
\hline Exaustão emocional & 0,07 & 0,07 & 0,17 & 0,22 & 0,18 & 0,001 \\
\hline Sobrecarga & 0,09 & 0,02 & 0,12 & 0,12 & 0,10 & 0,04 \\
\hline \multicolumn{7}{|l|}{ Manejo } \\
\hline Suporte social & 0,03 & 0,03 & 0,15 & 0,17 & 0,16 & 0,003 \\
\hline
\end{tabular}

$B$ indica os coeficientes de regressão não-padronizados no último passo da análise, quando cada termo foi corrigido para todos os outros do modelo. $* p<0,01 * * p<0,001$

\section{Discussão}

Os resultados mostraram características psicométricas promissoras para a versão brasileira da escala de coping em ambiente ocupacional. Assim como o instrumento original, a escala brasileira reforçou o modelo teórico que sustenta estilos gerais de coping envolvendo cognições e intenções de comportamentos de controle, de conteúdo escapista ou de manejo de sintomas, com índices de consistência interna considerados satisfatórios e superiores aos da escala original.

O padrão das relações entre as variáveis do estudo e as dimensões de coping corrobora os resultados de outros estudos, acrescentando evidências de validade de critério à escala traduzida. A independência de influências demográficas, por exemplo, foi um resultado encontrado por Amirkhan (1990), no artigo em que apresentou a CSI. Aquele autor afirmou que tal independência é desejável, pois dispensa a necessidade de se realizar ajustes nas normas populacionais, ao mesmo tempo em que ressaltou que poucas escalas de coping apresentam tal característica.

A associação entre demandas ocupacionais e estratégias de coping replica, em parte, resultados de outros estudos. Autores têm apresentado associações positivas, tais como as apresentadas neste trabalho, entre suporte social e controle (Amirkhan, 1990; Folkman \& cols., 1986; Latack, 1986), bem como fracas associações entre suporte social e esquiva (Amirkhan, 1990; Latack, 1986). Latack destacou que a associação positiva entre controle e suporte social se deve ao fato de que suporte tem um papel maior do que o de simples apoio emocional, estando associado ao engajamento dos indivíduos em estratégias proativas de controle, diante de situações de estresse.

Relações indiretas entre demandas ocupacionais e as estratégias de coping têm sido muito pouco exploradas. Parkes (1986) procurou evidências da interação entre variáveis individuais, fatores ambientais e características situacionais, interação esta prevista na própria definição de coping de acordo com a teoria transacional do estresse (Lazarus \& Folkman, 1984, citados por Parkes, 1986). Utilizando análises de regressão hierárquica, seus resultados apoiaram a hipótese transacional: o efeito direto das variáveis independentes não foi verificado, enquanto que os módulos interativos foram preditores significativos dos escores de coping. Em seu trabalho, Parkes observou a interação entre uma medida de neuroticismo e sobrecarga quadrática na predição de escores de controle. Neste estudo, exaustão emocional foi também moderador da relação entre sobrecarga quadrática e controle ( $p=0,03)$, conforme verificado pela inclusão do módulo interativo ao final da análise de regressão hierárquica, após a entrada de todas as variáveis envolvidas na interação. As relações diretas encontradas neste estudo entre demandas no trabalho e estratégias de coping não corresponderam aos achados de Parkes. Não foram encontrados outros estudos que tivessem por objetivo avaliar preditores dos estilos de coping. Por esta razão, especula-se que as diferenças aqui relatadas se devam a questões de ordem metodológica, como as diferentes medidas utilizadas nos dois estudos, ou a características inerentes às amostras.

Latack (1986) apresentou resultados semelhantes aos encontrados neste estudo, relativamente à relação entre esquiva, controle e exaustão emocional, embora a medida de reatividade ao estresse escolhida tenha sido uma escala de ansiedade. Como no presente estudo, controle esteve associado negativamente aos escores de ansiedade, enquanto que esquiva mostrou associação positiva. Amirkhan (1990), em sua amostra de 357 sujeitos de diferentes ocupações, também relatou a associação positiva entre escores em uma escala de depressão e esquiva, e a associação negativa entre os escores de depressão e controle (conforme mensurados por subescalas da CSI). Estes resultados fornecem sustentação à hipótese de que indivíduos que adotam estratégia de controle estão menos propensos a relatar ansiedade relacionada ao trabalho, enquanto aqueles que utilizam preferencialmente estratégias de esquiva ou de manejo de sintomas sejam mais propensos a relatar queixas psicossomáticas (Latack, 1986). Conclusões acerca de relações causais e mecanismos de retroalimentação não podem ser derivadas das análises apresentadas neste e nos estudos relatados, por limitações metodológicas. Porém, é razoável supor que a utilização de estratégias de controle esteja associada positivamente à percepção favorável do ambiente de trabalho e negativamente ao estresse, enquanto que sob circunstâncias insalubres como a sobrecarga de trabalho ou sob exaustão emocional intensa, esquiva seja a estratégia mais utilizada, e não necessariamente a mais eficaz. É conveniente 
lembrar da afirmação de Dewe e cols. (1993), ressaltando a importância dos dois processos de avaliação cognitiva envolvidos no coping: (1) a avaliação primária, onde o indivíduo avalia se há estresse e (2) a avaliação secundária, onde se avaliam os recursos e estratégias disponíveis. Considerando as restrições impostas pelo ambiente ocupacional, esquiva pode ser a mais adaptativa estratégia disponível em um contexto altamente estressante.

Finalmente, é importante que sejam consideradas as limitações inerentes a estudos transversais como este, cujos resultados baseiam-se apenas em instrumentos de autopreenchimento. Estudos posteriores poderão investigar a replicação dos resultados em ocupações específicas, assim como a estabilidade da estrutura apresentada para a versão brasileira da escala de coping ocupacional.

\section{Referências}

Amirkhan, J.H. (1990). A factor analytically derived measure of coping: The coping strategy indicator. Journal of Personality and Social Psychology, 59, 1066-1075.

Carver, C.S., Scheier, M.F. \& Weintraub, J.K. (1989). Assessing coping strategies: A theoretically based approach. Journal of Personality and Social Psychology, 45, 267-283.

Cohen, F. (1987). Measurement of coping. Em Kasl S.V. \& Cooper, C.L. (Eds.), Research methods in stress and health psychology (pp. 283-305). New York: John Wiley \& Sons.

Dewe, P., Cox, T. \& Ferguson, E. (1993). Individual strategies for coping with stress at work: a review. Work \& Stress, 7(1), 5-15.

Endler, N.S. \& Parker, J.D.A. (1990). Multidimensional assessment of coping: a critical evaluation. Journal of Personality and Social Psychology, 58(5), 844-854.

Folkman, S., Lazarus R.S. (1980). An analysis of coping in a middle-aged community sample. Journal of Health and Social Behavior, 25, 229-244.

Folkman, S., Lazarus, R.S., Dunkel-Schetter, C., DeLongis, A. \& Gruen, R.J. (1986). Dynamics of a stressful encounter: cognitive appraisal, coping and encounter outcomes. Journal of Personality and Social Psychology, 50(5), 992-1003.
Gaines, J. \& Jermier, J.M. (1983). Emotional exhaustion in a high stress organization. Academy of Management Journal, 26(4), 567-586.

Latack, J.C. (1986). Coping with job stress: measures and future directions for scale development. Journal of Applied Psychology, 71(3), 377-385.

Lazarus R.S. \& Folkman, S. (1984). Coping and Adaptation. Em Gentry, W.D. (Ed), Handbook of Behavioral Medicine (pp. 282-325). New York: The Guilford Press

Parkes, K.R. (1984). Locus of control, cognitive appraisal and coping in stressful episodes. Journal of Personality and Social Psychology, 46(3), 655-668.

Parkes, K.R. (1986). Coping in stressful episodes: the role of individual differences, environmental factors, and situational characteristics. Journal of Personality and Social Psychology, 51(6), 1277-1292.

Parkes, K.R. (1990). Coping, negative affectivity, and the work environment: additive and interactive predictors of mental health. Journal of Applied Psychology, 75(4), 399-409.

Parkes, K.R. (1994). Personality and coping as moderator of work stress processes: models, methods and measures. Work \& Stress, 8(2), 110-129.

Pasquali, L. (1999). Análise fatorial: um manual teórico-prático. Brasília: Editora UnB.

Pinheiro, F.A. \& Tróccoli, B.T. (2001). Além dos fatores físicos: preditores psicossociais das doenças osteomusculares relacionadas ao trabalho. (Resumo) Em Resumos de Comunicações Científicas (cd-rom), II Congresso Norte Nordeste de Psicologia, Salvador, BA.

Tabachnick, B.G. \& Fidell, L.S. (2001). Using Multivariate Statistics. $3^{\text {rd }}$. edition. New York: Harper Collins.

Tamayo, M.R. \& Tróccoli, B.T. (2000). Construção e validação de uma versão modificada da subescala de exaustão emocional: reinterpretando o construto de burnout. (Resumo) Em Programa da 52 Reunião Anual da SBPC, pp. 95, Brasília, DF

Taylor, S.E. (1986). Health Psychology. New York: Random House. 